\title{
Amélioration de la modélisation couplée houle-courant-sédiment
}

Katell Guizien

Postdoctorante, Instituto do Mar, Université de Coimbra,3049 Coimbra

Codex, Portugal.

actuellement, ATER, LEGI, BP 53, 38041 Grenoble Cedex 9, France.

e-mail:guizien@hmg.inpg.fr

Paulo Silva

Enseignant, Département de Physique, Université d'Aveiro, 3810 Aveiro, Portugal.

\section{Résumé}

Le débit sédimentaire total prédit par un modèle de couche limite turbulente de type $\mathrm{k}-\mathrm{L}$ se compare favorablement aux expériences avec des sables moyens $\left(d_{50}=0.21 \mathrm{~mm}\right)$ sur fond plat mais dans le détail de la colonne d'eau, des divergences apparaissent. La concentration moyenne près du fond ("sheet flow") était sous estimée dans le modèle et la concentration instantanée dans la couche de suspension était mal décrite. La modélisation de l'entravement dans la couche de "sheet flow" permet de mieux décrire la concentration moyenne près du fond pour des sables fins à grossiers ( $d_{50}$ variant de $0.13 \mathrm{~mm}$ à $0.32 \mathrm{~mm})$.

\section{Abstract}

Total sediment load predicted using a $\mathrm{k}-\mathrm{L}$ turbulent boundary layer model agrees with measurements for sand with $d_{50}=0.21 \mathrm{~mm}$ under sheet flow conditions but discrepancies arise when looking in details of the water column. The mean concentration was underestimated by the model, whereas upper in the suspension layer, the phase shift between concentration and velocities time series is not reproduced by the model. Taking into account hindering in the sheet flow layer enables us to improve the mean concentration predictions near the bottom for sand with $d_{50}$ ranging from $0.13 \mathrm{~mm}$ to $0.32 \mathrm{~mm}$.

\section{Introduction}

Le transport sédimentaire est l'une des préoccupations majeures et prélimi-naires à tout aménagement du littoral. En effet, une modification même faible du transport de sédiment peut à long terme entraîner de profonds et durables changements de la morphologie cotiêre. Or la modélisation du transport de sédiment n'est pas encore un domaine bien maîtrisé. Depuis une quinzaine d'années, sous l'impulsion de la CEE, les chercheurs européens collaborent afin d'aboutir à des outils prévisionnels acceptables. C'est l'un des buts du projet SEDiment transport MOdelling in marine Coastal environment. A la base de ces outils pour la prévision, il y a une meilleure compréhension des processus et en particulier grâce à la modélisation de la couche limite de fond, 
le plus souvent turbulente en nature, sous un écoulement oscillatoire. Parmi les premiers modèles, Fredsoe (1984), à la suite de Bakker (1974), suppose à chaque instant un profil logarithmique de la vitesse dans la couche limite et en déduit la viscosité turbulente. Cette approche constitue la base du modèle Sediment Transport Program (STP). Huynh-Than and Temperville (1990) adaptent le modèle numérique de couche limite turbulente de type $\mathrm{k}-\mathrm{L}$ de Lewellen (1977) pour l'étude des écoulements oscillatoires avec courant. La modélisation du transport en suspension des sédiments non cohésifs a été introduite dans ce modèle par Tran Thu (1995) à travers le couplage sur la densité. Le degré de complexité des modèles numériques développés par la suite se situe entre les deux précédents. Parmi ces modèles, le modèle de la longueur de mélange (zéro équation) de Ribberink et Al Salem (1995) et le modèle à une équation de Li and Davies (1996) ont été intercomparés avec les deux précédents. De façon globale (débit sédimentaire total), les modèles STP et k-L se comparent favorablement aux expériences mais dans le détail de la colonne d'eau, des divergences apparaissent (Davies et al., 1995). En particulier, pour les écoulements sur fond plat (condition de "sheet flow"), des expériences pour un sable moyen de diamètre $d_{50}=0.21 \mathrm{~mm}$ et sous différentes conditions hydrodynamiques (Katapodi et al., 1994) ont permis de montrer que la concentration moyenne près du fond et donc le débit sédimentaire dans la couche de "sheet flow" étaient sous estimés par tous les modèles cités cidessus. Par contre, dans la couche en suspension la concentration moyenne est assez bien décrite voire très bien par le modèle $\mathrm{k}-\mathrm{L}$, mais la concentration instantanée dans cette couche était mal décrite. En effet, expérimentalement, on observe un déphasage progressif des pics de la concentration instantanée par rapport à la vitesse sur la colonne d'eau qui n'est pas reproduit par les modèles, induisant un débit nettement surestimé par rapport aux expériences dans la couche en suspension. Néanmoins, dans des conditions de "sheet flow", la majeure part du débit sédimentaire a lieu dans une couche restreinte proche du fond, environ $80 \%$ à $90 \%$ du débit total d'après Dohmen-Janssen (1998). C'est pourquoi la modélisation correcte de cette zone est cruciale. Récemment, des investigations expérimentales complémentaires menées par Sumer et al. (1996) montrent que le transport dans cette zone de "sheet flow", qui n'est pas décrit correctement par les modèles de charriage, est affecté par la turbulence de l'écoulement et que la concentration en sédiment y varie linéairement en fonction de la distance au fond. Par ailleurs, l'épaisseur de la couche de "sheet flow" augmente quand le paramètre de Shields augmente. Janssen et al. (1997) puis Dohmen-Janssen (1999) ont testé l'influence de la taille du sédiment pour des écoulements de "sheet flow". En particulier, plus le sédiment est fin, plus la zone de "sheet flow" est épaisse. Après avoir rappelé dans la section 2 les équations du modèle $\mathrm{k}-\mathrm{L}$ couplé, on présente dans la section 3 une modification du modèle de transport en suspension visant à mieux décrire la couche de "sheet flow". Cette approche constitue une alternative aux modèles diphasiques tel que celui proposé par Dong and Zhang (1999). 


\section{Le modèle numérique de couche limite turbulente}

La partie hydrodynamique du modèle IDV de l'interaction houle-courantsédiment est basée sur le modèle numérique de couche limite turbulente dévelop-pé par Huynh Than et Temperville (1990). Il s'agit d'un modèle à deux équations de fermeture de type $\mathrm{k}-\mathrm{L}$ dans lequel le couplage avec le sédiment a été introduit par Tran Thu (1995) à travers une stratification verticale. Les équations à résoudre dans la couche limite de la version couplée sont alors les suivantes :

$$
\begin{aligned}
\frac{\partial u}{\partial t}= & \frac{\partial U}{\partial t}+\frac{\partial}{\partial z}\left(\nu_{t} \frac{\partial u}{\partial z}\right) \\
\frac{\partial v}{\partial t}= & \frac{\partial V}{\partial t}+\frac{\partial}{\partial z}\left(\nu_{t} \frac{\partial v}{\partial z}\right) \\
\frac{\partial k}{\partial t}= & \nu_{t}\left[\left(\frac{\partial u}{\partial z}\right)^{2}+\left(\frac{\partial v}{\partial z}\right)^{2}\right]-\frac{\sqrt{2 k}}{4 L} k+0.3 \frac{\partial}{\partial z}\left(\sqrt{2 k} L \frac{\partial k}{\partial z}\right)+\frac{g}{\rho_{0}} \gamma_{t} \frac{\partial \rho}{\partial z}(3) \\
\frac{\partial L}{\partial t}= & -0.175 \frac{\nu_{t}}{k}\left[\left(\frac{\partial u}{\partial z}\right)^{2}+\left(\frac{\partial v}{\partial z}\right)^{2}\right] L-\frac{0.375}{\sqrt{k}}\left(\frac{\partial(\sqrt{2 k} L)}{\partial z}\right)^{2} \\
& +0.075 \sqrt{2 k}+0.3 \frac{\partial}{\partial z}\left(\sqrt{2 k} L \frac{\partial L}{\partial z}\right)+0.8 \frac{L}{2 k} \frac{g}{\rho_{0}} \gamma_{t} \frac{\partial \rho}{\partial z} \\
\nu_{t}= & \frac{\sqrt{2 k}}{4} L \frac{1-16.444 \Omega}{(1-19.778 \Omega)(1-\Omega)} \\
\gamma_{t}= & \frac{4}{3} \nu_{t} \frac{1-\Omega}{(1-16.444 \Omega)} \\
\Omega= & \frac{2}{3} \frac{g}{\rho_{0}} \frac{d \rho}{d z} \frac{L^{2}}{k}
\end{aligned}
$$

où $u, v, U, V$ sont respectivment les vitesses horizontales et verticales dans la couche limite et dans l'écoulement externe, $\rho=\rho_{0}+C\left(\rho_{s}-\rho_{0}\right)$ décrit la stratification verticale dûe au mélange fluide-sédiment. On impose les conditions limites suivantes au fond et au sommet de la couche limite :

- en $z=z_{0}: u=0, v=0, \partial k / \partial z=0$ et $L=0.67 z_{0}$ d'après la solution analytique pour les écoulements permanents obtenue en supposant que production égale dissipation (équation de $k$ ) et un profil logarithmique de vitesse comme loi de paroi.

- en $z=z_{h}: u=U, v=V$ (raccordement avec l'écoulement externe); sans courant $k=0$ et $L=0$ car l'écoulement externe est potentiel, avec du courant $\partial k / \partial z=0$ et $\partial L / \partial z=0$ qui donne des résultats proches de la solution analytique pour les écoulements permanents.

Le transport du sédiment est décrit par l'équation de bilan de la concen- 
tration :

$$
\frac{\partial C}{\partial t}=\frac{\partial\left(w_{s} C\right)}{\partial z}+\frac{\partial}{\partial z}\left(\gamma_{t} \frac{\partial C}{\partial z}\right)
$$

où $w_{s}$ est la vitesse de chute du sédiment et avec les conditions limites suivantes:

- en $z=z_{0}: C=\max \left(C_{1}, C_{2}\right)$ où $C_{1}$ est la concentration de référence déterminée par la formule de Engelund et Fredsoe (1976) et $C_{2}$ est une concentration résultant de la chute des particules en suspension dans les couches supérieures.

- en $z=z_{h}$ : la concentration à ce niveau résulte du bilan entre la chute du sédiment et la diffusion turbulente si l'on suppose un flux nul à la suirface, soit à résoudre $w_{s} C+\gamma_{t} \partial C / \partial z=0$.

Le système d'équations (1)-(9) constitue la base de la modélisation numérique du couplage houle-courant-sédiment. Cependant, cette modélisation n'est pas complète car la vitesse de chute locale $w_{s}$ du sédiment n'est pas connue. Jusqu'à présent celle-ci a toujours été supposée constante, égale à la vitesse de chute d'une particule isolée dont le diamètre correspond au diamòtre $d_{50}$ du sédiment considéré. La partie hydrodynamique de ce modèle a été validée sous des conditions de houle régulière avec ou sans courant par comparaison avec des expériences dans le régime turbulent rugueux (Huynh Than and Temperville, 1990). Mais pour ce qui concerne la partie sédimentaire, si de façon globale, le débit net de sédiment dans la couche limite de fond était en bon accord avec celui mesuré dans des expériences sous des conditions de houle régulière en "sheet flow", dans le détail de la colonne d'eau, un certain nombre de déficiences apparaissent (Davies et al., 1995). Principalement, la concentration dans la couche de "sheet flow" est sous-estimée en moyenne et la concentration instantanée dans la couche en suspension est déphasée par rapport à celle mesurée.

\section{Prise en compte de la zone de "sheet flow"}

Partant des observations expérimentales, la terminologie "sheet flow" a été introduite pour désigner les expériences où les rides étaient absentes (fond plat). Les rides semblent en effet balayées par le déplacement horizontal des particules qui est important au fond. Ces expériences se caractérisent également par des concentrations très élevées dans la zone proche du fond. Fredsoe définit d'ailleurs l'épaisseur de la zone de "sheet flow" à l'aide d'un critère sur la concentration moyenne tel que $\langle C\rangle \geq 26.5 \mathrm{~g} / \mathrm{l}$ soit $1 \%$ en volume. DohmenJanssen (1999) a étudié de façon systématique l'épaisseur de la couche de "sheet flow" sous différentes conditions de houles régulières avec courant et pour différents diamètres de sable. L'épaisseur de la couche de "sheet flow" est définie à l'aide du critère $\langle C\rangle \geq 200 \mathrm{~g} / \mathrm{l}$, en s'appuyant sur le fait que pour une concentration de $8 \%$ en volume, la distance séparant deux particules est en moyenne égale à un diamètre de grain. Ceci suggère que l'entravement dans cette zone doit être important. Or, il a déjà été montré que la vitesse de chute d'une particule entravée est plus faible que celle d'une particule isolée. 

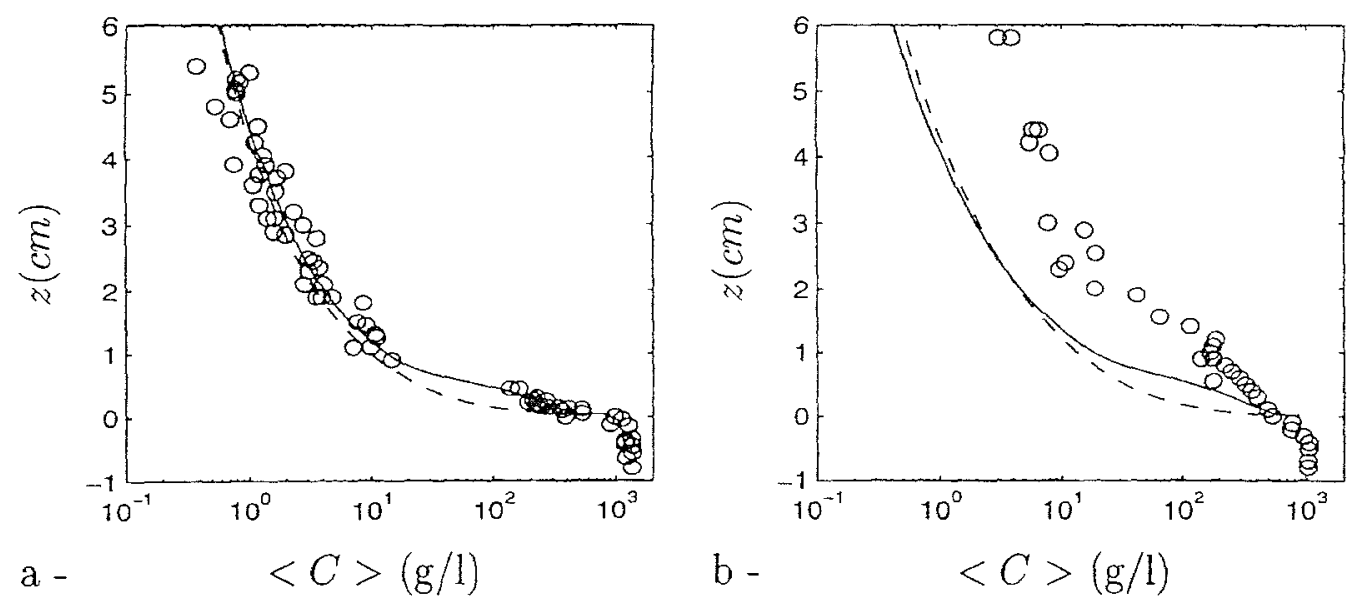

FIG. 1 - Profils verticaux de la concentration moyenne mesurés (0) et obtenus numériquement avec la modification de la vitesse de chute [critère de 1\%] (-) ou sans (- ) : (a) $d_{50}=0.21 \mathrm{~mm}$ (b) $d_{50}=0.13 \mathrm{~mm}$.

FIG. 1 - Mean concentration vertical profiles measured (o) and computed with settling velocity modification [1\% criterion] (-) or without it (- -) : (a) $d_{50}=$ $0.21 \mathrm{~mm}(b) d_{50}=0.13 \mathrm{~mm}$.

La vitesse de chute peut être alors modélisée d'après Lewis et al. (1949) par $w_{s}=w_{s 0}(1-C)^{n}$ où $C$ est la concentration, $w_{s 0}$ est la vitesse de chute d'une particule isolée et $n$ est un exposant. Richardson and Zaki (1954) ont montré par analyse dimensionnelle que $n$ est fonction du nombre de Reynolds de grain $R=w_{s 0} d_{50} / \nu$ où $\nu$ est la viscosité du fluide et est généralement de l'ordre de 2.5-4.5. Récemment Cheng (1997) introduit dans l'expression de $n$ une dépendance en fonction de la concentration mais qui ne modifie pas franchement son ordre de grandeur.

Or, dans la couche de "sheet flow", la prise en compte de l'entravement dans la vitesse de chute n'est pas suffisante. En effet, dans cette zone, la concentration est si importante et la vitesse horizontale si forte que les particules ne semblent quasiment ne plus chuter. L'expression de "sheet flow" vient d'ailleurs de l'observation de cette couche où les particules donnent l'impression de se déplacer dans des couches superposées. Ainsi, afin de modéliser cette zone, on se propose d'y imposer une vitesse de chute quasiment nulle, correspondant à un entravement maximum. Numériquement, cette condition se traduit par $w_{s}=w_{s 0}(1-C)^{20}$ tant que $\langle C\rangle \geq 1 \%$ ou $8 \%$. Pratiquement, on initialise le calcul avec la vitesse de chute d'une particule isolée puis on cor-. rige la vitesse de chute en installant le critère de la couche de "sheet flow" à chaque itération. La convergence vers une épaisseur constante de la couche de "sheet flow" est assez rapide (10 itérations au maximum). Sur les figures 1 , on représente le profil vertical de la concentration moyenne mesuré par DohmenJanssen comparé à celui obtenu à l'aide du modèle numérique avec et sans prise en compte de la zone de "sheet flow" pour les mêmes conditions que celles de l'expérience. 
La modification de la vitesse de chute dans la zone de "sheet flow" a effectivement permis de reproduire la concavité de la courbe de concentration moyenne que l'on observe sur les mesures expérimentales. Dans le tableau 2, on reporte les épaisseurs de la couche de "sheet flow" obtenues par DohmenJanssen expérimentalement avec le critère de $8 \%$ pour différentes conditions expérimentales ainsi que les épaisseurs obtenues par la modélisation numérique en utilisant les deux critères de $1 \%$ et $8 \%$ dans les mêmes conditions. Les conditions des expériences sont détaillées dans le tableau 1.

\begin{tabular}{|c|c|c|c|c|}
\hline $\mathrm{N}^{o}$ & $d_{50}(\mathrm{~mm})$ & $\begin{array}{l}\text { courant } \\
u_{c}(\mathrm{~m} / \mathrm{s})\end{array}$ & $\begin{array}{c}\text { houle } \\
u_{h}(\mathrm{~m} / \mathrm{s})\end{array}$ & $\begin{array}{c}\text { période } \\
T(\mathrm{~s})\end{array}$ \\
\hline D1 & 0.13 & 0.24 & 1.47 & 7.2 \\
\hline DQ & 0.21 & 0.24 & 1.47 & 7.2 \\
\hline$D 3$ & 0.32 & 0.24 & 1.47 & 7.2 \\
\hline U2 & 0.13 & 0.43 & 0.94 & 7.2 \\
\hline$T 1$ & 0.13 & 0.24 & 1.1 & 4 \\
\hline$T^{1} 2$ & 0.13 & 0.24 & 1.1 & 12 \\
\hline
\end{tabular}

TAB. 1 - Conditions expérimentales de Dohmen-Janssen (1999) noté DJ99 TAB. 1 - Experimental conditions from Dohmen-Janssen (1999) denoted by DJ99.

On reporte égailement dans le tableau 2 entre parenthèses le niveau où la concentration moyenne vaut $8 \%$ pour le critère limite de $1 \%$. Il est à noter que l'épaisseur de la couche de "sheet flow" est définie par Dohmen-Janssen comme la distance entre le niveau où la concentration moyenne est de $8 \%$ et le niveau où les grains sont immobiles pour le maximum de vitesse. Or ce niveau se situe 3 à $4 \mathrm{~mm}$ sous le niveau $z=0$.

\begin{tabular}{cccc} 
Expériences & DJ 99 & \multicolumn{2}{c}{$\begin{array}{c}\text { modèle k-L avec } \\
<C>\geq 8 \% \\
<C>\geq 1 \%\end{array}$} \\
& $\delta_{s}(\mathrm{~mm})$ & $\delta_{s}(\mathrm{~mm})$ & $\delta_{s}(\mathrm{~mm})$ \\
\hline$D 1$ & 12 & 1.7 & $9.0(4.0)$ \\
$D 2$ & 6 & 1.2 & $6.9(2.6)$ \\
D3 & 5 & 1.0 & $5.3(2.0)$ \\
U2 & 5 & 0.5 & $3.8(0.8)$ \\
T1 & 6 & 1.0 & $5.0(2.1)$ \\
T2 & 8 & 0.6 & $4.3(1.3)$ \\
\hline
\end{tabular}

TAB. 2 - Epaisseur de la couche de "sheet flow" en mm pour différentes conditions expérimentales comparées aux résultats du modèle $\mathrm{k}-\mathrm{L}$ pour les deux critères

TAB. 2 - Sheet flow layer thickness in $\mathrm{mm}$ for different expriments conditions compared with computations from $k-L$ model with both criteria.

Il est remarquable que les tendances observées expérimentalement sont re- 
produites par le modèle numérique pour les deux critères. En effet, l'épaisseur de la couche de "sheet flow" diminue lorsque le diamètre du grain augmente et qu'elle dépend assez peu de la période de la houle mais bien plus de son amplitude et de celle du courant. Quantitativement, les épaisseurs sont assez bien prédites pour les sables les plus grossiers $\left(d_{50}=0.21 \mathrm{~mm}\right.$ et $\left.d_{50}=0.32 \mathrm{~mm}\right)$ avec le critère d'entravement tant que la concentration moyenne est supérieure à $1 \%$ en volume. Pour le sable le plus fin, l'épaisseur de la couche de "sheet flow" mais également la concentration moyenne sont encore sous-estimés par le modèle modifié. On peut également comparer le débit sédimentaire qui s'en déduit dans la couche de "sheet flow" en intégrant le débit du fond jusqu'au niveau où la concentration moyenne vaut $1 \%$, comme le propose DohmenJanssen (table 3).

\begin{tabular}{|c|c|c|c|}
\hline Expériences & $\begin{array}{c}\text { DJ } 99 \\
q_{s}\left(10^{-6} \mathrm{~m}^{2} / \mathrm{s}\right)\end{array}$ & $\begin{array}{c}\text { modèle } \mathrm{k}-\mathrm{L} \text { avec } \\
\text { entravement } \mathrm{si}<C>\geq 1 \% \\
q_{s}\left(10^{-6} \mathrm{~m}^{2} / \mathrm{s}\right)\end{array}$ & $\begin{array}{c}\text { modèle } \mathrm{k}-\mathrm{L} \\
\text { non modifié } \\
q_{s}\left(10^{-6} \mathrm{~m}^{2} / \mathrm{s}\right)\end{array}$ \\
\hline D1 & 55.2 & 127.1 & 49.3 \\
\hline D2 & 102.0 & 130.8 & 40.0 \\
\hline D3 & 77.6 & 94.5 & 24.2 \\
\hline $\mathrm{U} 2$ & 72.1 & 89.6 & 27.5 \\
\hline
\end{tabular}

TAB. 3 - Débit sédimentaire dans la couche de "sheet flow" pour différentes conditions expérimentales comparées aux résultats du modèle $k-L$ avec ou sans modification

ТАВ. 3 - Sediment load in the sheet flow layer from experiments and computations with $k$ - $L$ model before and after modification

Comme pour la concentration moyenne, en prenant en compte l'entravement iorsque la concentration est élevée, on améliore l'accord avec les expériences pour les sédiments moyens à gros mais pas franchement pour le sédiment fin. C'est sur le détail des séries temporelles de la concentration près du fond sur les figures 2 que l'on observe l'effet de la modification de la vitesse de chute près du fond.

En effet, en prenant en compte l'entravement dans la couche de "sheet flow", on prédit mieux le niveau de la concentration aux instants du maximum de frottement de fond pour les différents sables. Mais aux instants près de la renverse de l'écoulement, les pics de la concentration sont très largement sous estimés par le modèle, surtout pour le sédiment le plus fin. Ces pics sont dûs à des pics secondaires de la diffusivité turbulente que le modèle $k-L$ ne reproduit pas. Or ces pics sont particulièrement importants et ne sont plus vraiment secondaires dans le cas des sédiments fins. D'après Savioli and Justesen (1996), il est possible d'obtenir ces pics dans un modèle $k-\epsilon$. Il est tout à fait envisageable de prendre en compte la modification de la vitesse de chute près du fond proposée ici dans d'autresmodèles de la couche limite turbulente de fond dans lesquels la modélisation de la diffusivité turbulente 
serait meilleure.
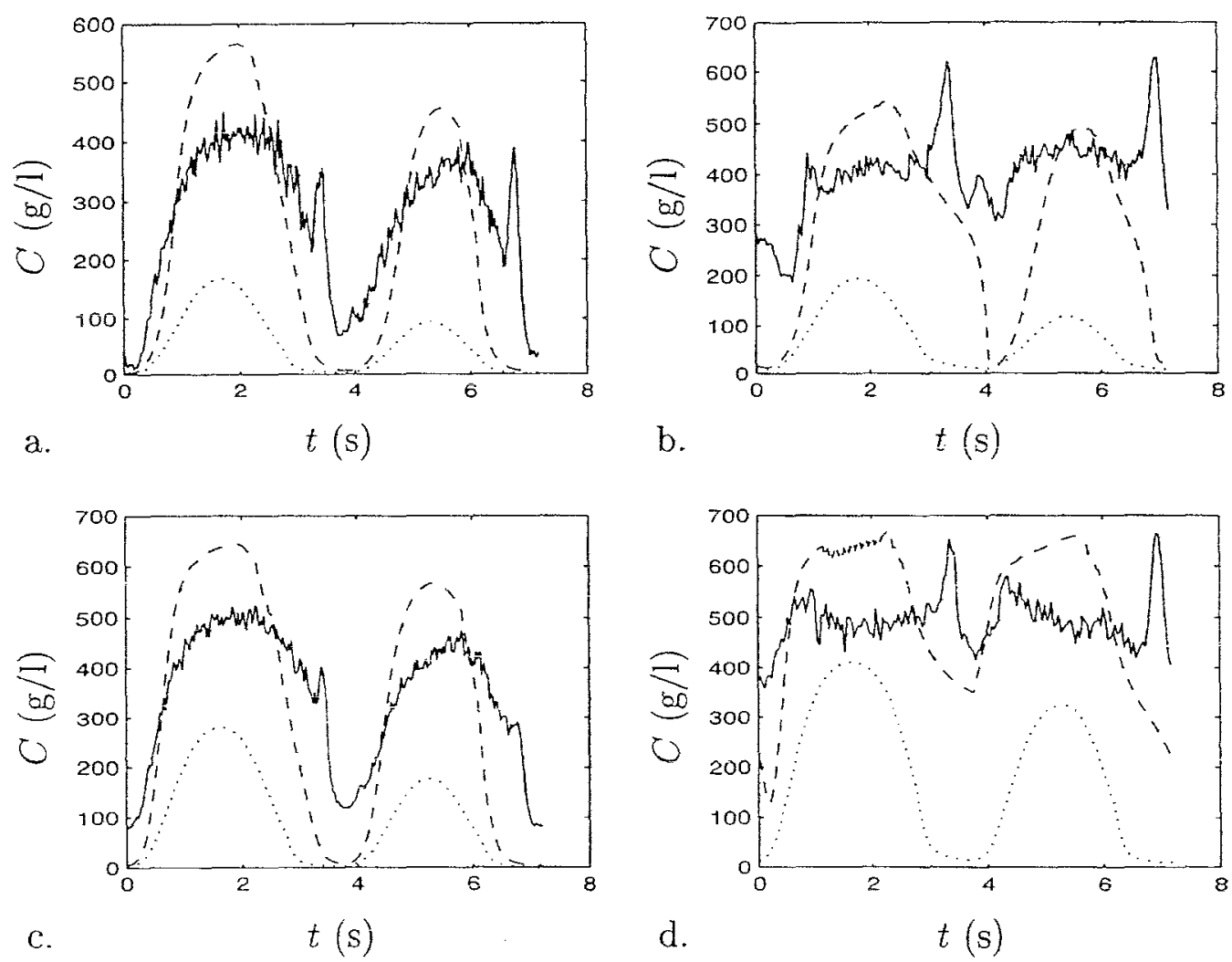

Fig. 2 - Séries temporelles de la concentration mesurée (-) et obtenus numériquement avec la modification de la vitesse de chute (--) ou sans ( . .) : pour $d_{50}=0.21 \mathrm{~mm}$ à $z=1.5 \mathrm{~mm}$ du fond (c) et à $z=2.5 \mathrm{~mm}$ du fond (a) et pour $d_{50}=0.13 \mathrm{~mm}$ à $z=1 \mathrm{~mm}$ du fond (d) et à $z=3 \mathrm{~mm}$ du fond (b). FIG. 2 - Concentration time series measured (-) and computed with (- ) and without ( $\cdots$ ) the settling velocity modification : for $d_{50}=0.21 \mathrm{~mm}$ at $z=1.5 \mathrm{~mm}$ (c) and at $z=2.5 \mathrm{~mm}$ from the bottom (a) and for $d_{50}=0.13 \mathrm{~mm}$ at $z=1 \mathrm{~mm}$ (d) and at $z=3 \mathrm{~mm}$ from the bottom (b).

\section{Conclusion}

La prise en compte de l'entravement au niveau où la concentration est très élevée, en moyenne supérieure à $1 \%$ en volume, dans un modèle de couche limite turbulente de fond de type $\mathrm{k}-\mathrm{L}$ a permis d'améliorer la prédiction du transport sédimentaire pour les sédiments moyens dans cette couche de quelques millimètres prés du fond. Des efforts sont cependant encore à fournir pour améliorer la modélisation de la diffusivité turbulente et de ses pics secondaires au moment de la renverse, particulièrement pour les sédiments fins.

\section{Remerciements}

Les auteurs remercient la CEE pour avoir financé ce travail dans le cadre 
du projet SEDMOC (contrat MAS3-CT97-0115). Le premier auteur remercie IMAR (Universidade de Coimbra - Portugal) de lui avoir accordé une bourse post-doctorale.

\section{Références}

BAKKER W.T. (1974) - Sand concentration in an oscillatory flow, Proc. of the $14^{\text {th }}$ Coastal Eng. Conf., ASCE, pp 1129-1148.

CHENG N.-S. (1997) - Effect of concentration on settling velocity of sediment particles, J. Hydraulic Eng., pp 728-731.

DAVIES A.G., RIBBERINK J.S., TEMPERVILLE A., VILLARET C., ZYSERMAN J.A. and EIDVISK K. (1995) - Intercomparison of models of wavecurrent-sediment interaction, abst. 2.4 MAST/2G8M, Final overall meeting, Gdansk (Poland).

DOHMEN-JANSSEN M. (1999) - Grain size influence on sediment transport in oscillatory sheet flow, PhD Thesis, Université Technique Delft.

DONG P. and ZHANG K. (1999) - Two-phase flow modelling of sediment motions in oscillatory sheet flow, Coastal Eng., 36, pp 87-109.

ENGELUND F. and FREDSOE J. (1976) - A sediment transport model for straight alluvial channel, Nordic Hydrology 7, pp 293-306.

FREDSOE J. (1984) - Turbulent boundary layer in wave-current interaction, J. Hydr. Eng., ASCE, 110, HY8, pp 1103-1120.

HUYNH-THAN S. and TEMPERVILLE A. (1990) - A numerical model of the rough turbulent boundary layer in combined wave and current interaction, in Proc. of the $22^{\text {th }}$ Coastal Eng. Conf., ASCE, p 853-866.

JANSSEN M., HASSAN W., WAL. R. and RIBBERINK J.S. (1997) - Grain size influence on sand transport mechanisms, Coastal Dynamics, pp 58-68.

KATAPODI I., RIBBERINK I.S., RUOL P. KOELEWIJN R., LODHAL C., LONGO S., CROSATO A. and WALLACE H. (1994) - Intra-wave sediment transport in an oscillatory flow superimposed on a mean current, Report $H^{-}$ 1684, Part III, Delft (The Netherlands).

LEWELLEN W.S. (1977) - Use of invariant modelling, in Handbook of turbulence, vol. 1, Plenum Publishing Corp., Ed. FROST W., pp237-280.

LEWIS W.K., GILLILAND E.R. and BAUER W.C. (1949) - Characteristics of fluidized particles, Industrial Eng. Chem., 41, pp 1104-1114. 
LI Z. and DAVIES A.G. (1996) - Towards predicting sediment transport in combined wave-current flow, J. Waterway, Port, Coastal and Ocean Eng., ASCE, vol. 122, 4, pp 157-164.

RIBBERINK J.S. and AL SALEM A.A. (1995) - Sheet flow and suspension of sand in oscillatory boundary layers, Coastal Eng., 25, pp 205-225.

RICHARDSON J.F. and ZAKI W.N. (1954) - Sedimentation and fluidisation: part I, Trans. Inst. Chem. Engrs, 32(1), pp 35-53.

SAVIOLI J. and JUSTESEN P. (1996) - Sediment in oscillatory flows over a plane bed, J. Hydr. Res., vol. 35, 2, pp 177-190.

SUMER B.M., KOZAKIEWICZ A., FREDSOE J. and DEIGAARD (1996) Velocity and concentiation profiles in sheet-flow layer of movable bed, J. Hydr. Eng., vol. 122, 10, pp 549-558.

TRAN THU T. (1995) - Modélisation numérique de l'interaction houle-courantsédiment, Thèse de doctorat, Université Joseph Fourier - Grenoble I. 